\title{
REVISED ORBIT AND TRANSIT EXCLUSION FOR HD 114762b
}

\author{
Stephen R. Kane ${ }^{1}$, Gregory W. Henry ${ }^{2}$, Diana Dragomir ${ }^{1,3}$, Debra A. Fischer ${ }^{4}$, Andrew W. Howard ${ }^{5,6}$, \\ XUESONG WANG ${ }^{7}$, AND JASON T. WRIGHT ${ }^{7,8}$ \\ ${ }^{1}$ NASA Exoplanet Science Institute, Caltech, MS 100-22, 770 South Wilson Avenue, Pasadena, CA 91125, USA; skane@ipac.caltech.edu \\ ${ }^{2}$ Center of Excellence in Information Systems, Tennessee State University, 3500 John A. Merritt Blvd., Box 9501, Nashville, TN 37209, USA \\ ${ }^{3}$ Department of Physics \& Astronomy, University of British Columbia, Vancouver, BC V6T1Z1, Canada \\ ${ }^{4}$ Department of Astronomy, Yale University, New Haven, CT 06511, USA \\ ${ }^{5}$ Department of Astronomy, University of California, Berkeley, CA 94720, USA \\ ${ }^{6}$ Space Sciences Laboratory, University of California, Berkeley, CA 94720, USA \\ ${ }^{7}$ Department of Astronomy and Astrophysics, Pennsylvania State University, 525 Davey Laboratory, University Park, PA 16802, USA \\ ${ }^{8}$ Center for Exoplanets \& Habitable Worlds, Pennsylvania State University, 525 Davey Laboratory, University Park, PA 16802, USA \\ Received 2011 May 1; accepted 2011 June 7; published 2011 June 22
}

\begin{abstract}
Transiting planets around bright stars have allowed the detailed follow-up and characterization of exoplanets, such as the study of exoplanetary atmospheres. The Transit Ephemeris Refinement and Monitoring Survey is refining the orbits of the known exoplanets to confirm or rule out both transit signatures and the presence of additional companions. Here we present results for the companion orbiting HD 114762 in an eccentric 84 day orbit. Radial velocity analysis performed on 19 years of Lick Observatory data constrain the uncertainty in the predicted time of mid-transit to $\sim 5 \mathrm{hr}$, which is less than the predicted one-half day transit duration. We find no evidence of additional companions in this system. New photometric observations with one of our Automated Photoelectric Telescopes at Fairborn Observatory taken during a revised transit time for companion b, along with 23 years of nightly automated observations, allow us to rule out on-time central transits to a limit of $\sim 0.001 \mathrm{mag}$. Early or late central transits are ruled out to a limit of $\sim 0.002 \mathrm{mag}$, and transits with half the duration of a central transit are ruled out to a limit of $\sim 0.003 \mathrm{mag}$.
\end{abstract}

Key words: planetary systems - stars: individual (HD 114762) - techniques: photometric - techniques: radial velocities

\section{INTRODUCTION}

The radial velocity (RV) method is still the dominant source of confirmed exoplanets, although the transit technique is rapidly producing planet detections. The advantage of transiting planets is that they allow additional characterization of the planet, namely the radius and hence the density. For planets transiting bright stars, atmospheric studies may be undertaken, as was the case for HD 189733b (Knutson et al. 2009b) and HD 149026b (Knutson et al. 2009a). However, most of the planets detected using transits orbit relatively faint host stars, making them inaccessible to such investigations. Transit detections are also biased toward short periods due to the geometric transit probability (Kane \& von Braun 2008). The purpose of the Transit Ephemeris Refinement and Monitoring Survey (TERMS) is to ameliorate these biases through photometric monitoring of known RV planets at improved times of predicted transits (Kane et al. 2009).

The first planet candidate to be detected with the RV technique orbits the star HD 114762, a late F dwarf. The companion was discovered by Latham et al. (1989) who reported the minimum mass as $0.011 \pm 0.001 M_{\odot}$. This discovery was confirmed by Cochran et al. (1991) and a search for transits by Robinson et al. (1990) ruled out transit depths greater than 0.01 mag. Hale (1995) used high-resolution spectroscopy to measure the projected rotational velocity $v \sin i$ and concluded that the inclination of the companion is likely to be low, possibly rendering the companion above the limit for deuterium fusion. There is still uncertainty as to the true inclination of the companion, particularly with the large range of spin-orbit misalignments that can occur (Fabrycky \& Winn 2009). Further long-term photometry by Henry et al. (1997) has found the host star to be photometrically stable in the $V$ band to 0.001 mag.

Here we present our complete RV data set from Lick Observatory that has a time baseline of 19 years. The Keplerian analyses of these data are used to produce a revised orbital solution that further refines the period for transit studies and rules out the presence of additional companions within the system. We also present 23 years of high-precision photometry from the $\mathrm{T} 2$ $0.25 \mathrm{~m}$ and the T10 $0.8 \mathrm{~m}$ Automatic Photoelectric Telescopes (APTs) at Fairborn Observatory. The most recent data were acquired with the T10 APT whose $1 \sigma$ uncertainty of $0.0012 \mathrm{mag}$ for a single observation is easily sufficient to find or to rule out transits with a predicted depth of $\sim 1 \%$.

\section{REVISED ORBITAL PARAMETERS}

The RV data were acquired with the $3.0 \mathrm{~m}$ Shane telescope and the Hamilton Echelle Spectrograph at Lick Observatory. The data comprise 74 measurements that range from 1990 March through 2009 February and are shown in Table 1. Of these, 46 were extracted from the same spectra as used by Butler et al. (2006). However, the Lick data reduction pipeline undergoes frequent refinement and so each reduction is of superior quality to previously published measurements extracted from those spectra. As data accumulates, the code adaptively reassesses the relative weights of portions of the spectra based upon factors such as contamination by weak telluric lines or bad pixels. The fourth column in Table 1 shows the dewar number which was used with the observation, the relevance being the different CCD response characteristics that can occur between dewars. This is accounted for in the Keplerian orbital fitting described below. 
Table 1

Lick Radial Velocities

\begin{tabular}{|c|c|c|c|}
\hline $\begin{array}{l}\text { Date } \\
(\mathrm{JD}-2440000)\end{array}$ & $\begin{array}{l}\text { Radial Velocity } \\
\qquad\left(\mathrm{m} \mathrm{s}^{-1}\right)\end{array}$ & $\begin{array}{l}\text { Uncertainty } \\
\qquad\left(\mathrm{m} \mathrm{s}^{-1}\right)\end{array}$ & Dewar \\
\hline 7964.963900 & -185.78 & 22.28 & 6 \\
\hline 8017.833900 & 315.12 & 20.41 & 6 \\
\hline 8018.840200 & 277.92 & 17.40 & 6 \\
\hline 8019.830500 & 289.41 & 27.20 & 6 \\
\hline 8375.852800 & -600.95 & 14.63 & 6 \\
\hline 8437.738000 & 262.91 & 19.82 & 6 \\
\hline 8649.091100 & 465.42 & 17.25 & 8 \\
\hline 8670.973032 & 569.49 & 21.00 & 8 \\
\hline 9068.820700 & 452.17 & 25.78 & 8 \\
\hline 9068.842500 & 505.42 & 30.66 & 8 \\
\hline 9096.814400 & 518.85 & 27.31 & 8 \\
\hline 9096.835900 & 532.71 & 28.77 & 8 \\
\hline 9114.776700 & 76.31 & 21.17 & 8 \\
\hline 9174.705300 & 573.39 & 14.70 & 8 \\
\hline 9350.008000 & 476.46 & 17.36 & 8 \\
\hline 9375.011500 & -296.95 & 17.90 & 8 \\
\hline 9464.786200 & -636.28 & 25.35 & 8 \\
\hline 9469.781200 & -554.16 & 16.03 & 8 \\
\hline 9768.932800 & 496.52 & 25.17 & 39 \\
\hline 9768.955078 & 513.54 & 22.34 & 39 \\
\hline 9801.853516 & -626.62 & 24.68 & 39 \\
\hline 9801.875000 & -602.62 & 28.81 & 39 \\
\hline 9801.899414 & -597.07 & 24.61 & 39 \\
\hline 9802.825195 & -535.91 & 23.30 & 39 \\
\hline 9802.848633 & -556.53 & 20.99 & 39 \\
\hline 9802.871094 & -585.50 & 23.00 & 39 \\
\hline 9803.818359 & -572.41 & 21.69 & 39 \\
\hline 9803.840820 & -600.02 & 22.14 & 39 \\
\hline 9803.863281 & -578.70 & 24.69 & 39 \\
\hline 9858.733398 & 409.48 & 25.35 & 39 \\
\hline 9881.718750 & -445.90 & 9.60 & 39 \\
\hline 9892.701172 & -304.06 & 24.17 & 39 \\
\hline 9892.723633 & -317.33 & 26.76 & 39 \\
\hline 9892.746094 & -336.66 & 27.65 & 39 \\
\hline 9893.698242 & -218.52 & 27.55 & 39 \\
\hline 9893.721680 & -271.28 & 24.85 & 39 \\
\hline 9893.744141 & -256.03 & 25.36 & 39 \\
\hline 9894.697266 & -237.50 & 20.05 & 39 \\
\hline 9894.719727 & -170.63 & 18.06 & 39 \\
\hline 9894.742188 & -203.70 & 19.28 & 39 \\
\hline 9913.755859 & 585.09 & 9.80 & 39 \\
\hline 9914.675781 & 608.46 & 9.31 & 39 \\
\hline 10072.061523 & 355.86 & 17.98 & 39 \\
\hline 10120.954102 & 151.07 & 23.88 & 39 \\
\hline 10120.976562 & 132.44 & 25.13 & 39 \\
\hline 10121.968750 & 96.44 & 23.06 & 39 \\
\hline 10121.990234 & 92.78 & 24.77 & 39 \\
\hline 10124.940430 & -7.39 & 23.47 & 39 \\
\hline 10124.962891 & 1.09 & 26.03 & 39 \\
\hline 10126.963867 & -144.75 & 26.88 & 39 \\
\hline 10126.984375 & -96.89 & 39.75 & 39 \\
\hline 10128.038086 & -257.56 & 24.87 & 39 \\
\hline 10128.062500 & -167.79 & 26.50 & 39 \\
\hline 10128.970703 & -245.66 & 22.94 & 39 \\
\hline 10128.994141 & -212.46 & 20.20 & 39 \\
\hline 10144.940430 & -335.55 & 11.58 & 39 \\
\hline 10172.857422 & 716.70 & 51.27 & 39 \\
\hline 10181.819336 & 583.34 & 14.87 & 39 \\
\hline 10187.833008 & 566.28 & 23.11 & 39 \\
\hline 10187.856445 & 520.18 & 26.32 & 39 \\
\hline 10200.783203 & 289.36 & 23.24 & 39 \\
\hline 10200.805664 & 284.61 & 22.44 & 39 \\
\hline 10504.960938 & 623.54 & 17.04 & 39 \\
\hline 11628.850586 & 197.24 & 13.89 & 18 \\
\hline 12033.864258 & 521.20 & 10.93 & 18 \\
\hline
\end{tabular}

Table 1

(Continued)

\begin{tabular}{lrcc}
\hline \hline $\begin{array}{l}\text { Date } \\
(\mathrm{JD}-2440000)\end{array}$ & $\begin{array}{c}\text { Radial Velocity } \\
\left(\mathrm{m} \mathrm{s}^{-1}\right)\end{array}$ & $\begin{array}{c}\text { Uncertainty } \\
\left(\mathrm{m} \mathrm{s}^{-1}\right)\end{array}$ & Dewar \\
\hline 12833.715820 & -71.10 & 9.78 & 24 \\
13068.975586 & -392.18 & 14.66 & 24 \\
13132.822266 & 373.24 & 10.72 & 24 \\
13389.043333 & 270.11 & 12.56 & 24 \\
13544.723507 & 501.86 & 10.65 & 24 \\
13545.745370 & 494.24 & 10.29 & 24 \\
14165.945718 & -625.59 & 11.30 & 24 \\
14547.955891 & 527.27 & 10.38 & 24 \\
14865.043218 & 576.89 & 13.83 & 24 \\
\hline
\end{tabular}

Table 2

Stellar Properties

\begin{tabular}{lc}
\hline \hline Parameter & Value \\
\hline$V$ & 7.3 \\
$B-V$ & 0.525 \\
Distance $(\mathrm{pc})$ & $38.7 \pm 1.1$ \\
$T_{\text {eff }}(\mathrm{K})$ & $5673 \pm 44$ \\
$\log g$ & $4.135 \pm 0.060$ \\
{$[\mathrm{Fe} / \mathrm{H}]$} & $-0.774 \pm 0.030$ \\
$v \sin i\left(\mathrm{~km} \mathrm{~s}^{-1}\right)$ & $1.77 \pm 0.50$ \\
$M_{\star}\left(M_{\odot}\right)$ & $0.83 \pm 0.01$ \\
$R_{\star}\left(R_{\odot}\right)$ & $1.24 \pm 0.05$ \\
\hline
\end{tabular}

We used Spectroscopy Made Easy (Valenti \& Piskunov 1996) to fit high-resolution Lick spectra of HD 114862, applying the wavelength intervals, line data, and methodology of Valenti \& Fischer (2005). We further constrained the surface gravity using Yonsei-Yale $\left(\mathrm{Y}^{2}\right)$ stellar structure models (Demarque et al. 2004) and revised Hipparcos parallaxes (van Leeuwen 2007) with the iterative method of Valenti et al. (2009). The resulting stellar parameters listed in Table 2 are effective temperature, surface gravity, iron abundance, projected rotational velocity, mass, and radius. The stellar radius, $R_{\star}=1.24 R_{\odot}$, is crucial for estimating the depth and duration of a planetary transit. These properties are consistent with a very metal-poor early-G sub-giant.

We fit a single-planet Keplerian orbital solution to the RV data using the techniques described in Howard et al. (2010) and the partially linearized, least-squares fitting procedure described in Wright \& Howard (2009). This was performed both with and without the inclusion of a linear trend in order to determine the significance of including that free parameter. The parameter uncertainties are extracted from the sampling distribution of each parameter through a non-parametric bootstrap analysis (Freedman 1981). Table 3 lists the fit parameters both with and without the trend.

As indicated by the $\chi_{\text {red }}^{2}$ and the rms scatter of the residuals, the Keplerian fit which includes the trend does not improve the fit given the addition of the free parameter. Thus, this trend is unlikely to be due to any additional companions within the system. This is consistent with the findings of Mugrauer et al. (2006) who exclude companions with masses greater than $66 M_{J}$ at orbital radii of 316-2674 AU. Note that Patience et al. (2002) detect a stellar companion to HD 114762, further characterized by Bowler et al. (2009), at an angular separation of 3"'3 (180 AU). This companion could feasibly cause the observed trend, but the significance level is too low for us to claim such a detection. We therefore adopt the solution without the trend included, shown in 


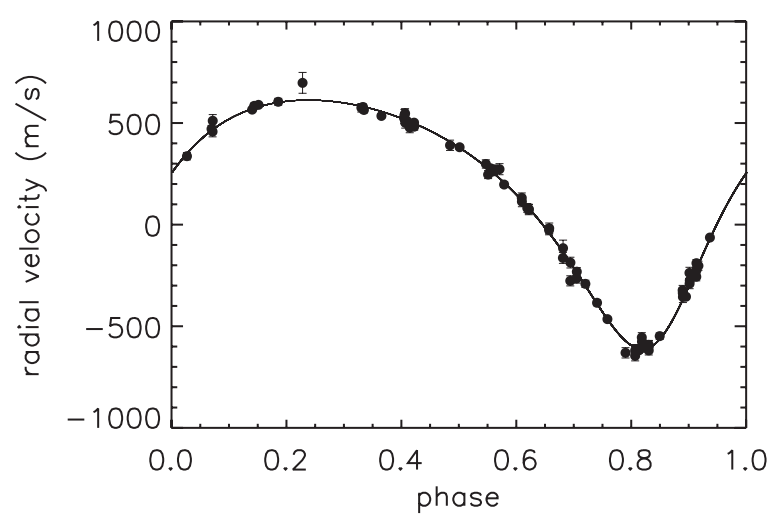

Figure 1. Radial velocity data of HD 114762 acquired with the $3.0 \mathrm{~m}$ Shane telescope at Lick Observatory. Also shown is the best-fit Keplerian model for the planet.

Table 3

Keplerian Fit Parameters

\begin{tabular}{lcc}
\hline \hline Parameter & Trend & No Trend \\
\hline$P($ days $)$ & $83.9152 \pm 0.0028$ & $83.9151 \pm 0.0030$ \\
$T_{c}{ }^{\mathrm{a}}(\mathrm{JD}-2440000)$ & $15326.595 \pm 0.242$ & $15326.665 \pm 0.242$ \\
$T_{p}{ }^{\mathrm{b}}(\mathrm{JD}-2440000)$ & $9889.141 \pm 0.190$ & $9889.106 \pm 0.186$ \\
$e$ & $0.3325 \pm 0.0048$ & $0.3354 \pm 0.0048$ \\
$K\left(\mathrm{~m} \mathrm{~s}^{-1}\right)$ & $612.72 \pm 3.35$ & $612.48 \pm 3.52$ \\
$\omega\left(\mathrm{deg}^{-1}\right.$ & $201.41 \pm 1.04$ & $201.28 \pm 1.01$ \\
$d v / d t\left(\mathrm{~m} \mathrm{~s}^{-1} \mathrm{yr}^{-1}\right)$ & $-3.83 \pm 1.91$ & $\mathrm{~N} / \mathrm{A}$ \\
$M_{p} \sin i\left(M_{J}\right)$ & $10.99 \pm 0.09$ & $10.98 \pm 0.09$ \\
$a(\mathrm{AU})$ & $0.353 \pm 0.001$ & $0.353 \pm 0.001$ \\
$\chi_{\text {red }}^{2}$ & 1.29 & 1.32 \\
$\mathrm{rms}\left(\mathrm{m} \mathrm{s}^{-1}\right)$ & 27.53 & 27.42 \\
\hline
\end{tabular}

Notes.

a Time of mid-transit.

b Time of periastron passage.

Figure 1. The fit required four additional free parameters due to the offsets induced by the changing of the detector dewars. The offsets with respect to the observations acquired with dewar 6 are $-6.81 \pm 5.88,19.31 \pm 6.26,17.42 \pm 8.91$, and $-5.40 \pm 7.12 \mathrm{~m} \mathrm{~s}^{-1}$ for dewars $8,39,18$, and 24 , respectively. The uncertainty in the period for the presented orbital solution is a factor of four improvement over the previous solution by Butler et al. (2006) and allows a more robust transit search to be carried out.

\section{TRANSIT EPHEMERIS REFINEMENT}

From the stellar and planetary properties listed in Tables 2 and 3, we derive a planetary radius of $R_{p}=1.11 R_{J}$ using the methods described in Bodenheimer et al. (2003). This results in a predicted transit duration of 0.49 days and a predicted transit depth of $0.91 \%$. The uncertainty in the stellar mass/ radius and subsequent uncertainty in the planetary mass/radius have a minor effect on the estimated transit duration but in no way affect the predicted transit mid-points since these are derived from the orbital parameters (Kane et al. 2009). Based upon the revised orbital parameters, we computed a new transit ephemeris, applied in the following section.

As described by Kane \& von Braun (2008), the probability of a planetary transit is a strong function of both the eccentricity and the argument of periastron and is at a maximum when the periastron passage occurs close to the star-observer line of sight $\left(\omega=90^{\circ}\right)$. The measured periastron argument for HD $114762 \mathrm{~b}$ leads to an orbital configuration which favors a secondary eclipse rather than a primary transit. The transit probability based upon the parameters of Table 3 is $1.71 \%$. For comparison, an equivalent circular orbit would have a transit probability of $1.73 \%$, but the same eccentricity with a periastron argument of $90^{\circ}$ would have a transit probability of $2.60 \%$.

\section{TRANSIT EXCLUSION AND IMPLICATIONS}

As part of our long-term program to measure luminosity cycles in solar-type stars (Henry 1999), we have acquired 23 consecutive years of photometric observations of HD 114762 with our T2 $0.25 \mathrm{~m}$ and T10 $0.80 \mathrm{~m}$ APTs at Fairborn Observatory in southern Arizona. The T2 APT used a photodiode detector to make differential measurements of stars through Johnson $V R I$ filters. It collected 1989 nightly observations of HD 114762 during the 1989 through 2001 observing seasons. Details concerning the data acquisition and reduction with this telescope can be found in Henry (1995) and Percy et al. (2000). The T10 APT has a two-channel photometer with a dichroic filter that separates the Stromgren $b$ and $y$ photometric bands so they can be measured simultaneously by two EMI 9124QB photomultiplier tubes. T10 collected 1137 nightly observations of HD 114762 during the 2002 through 2011 observing seasons. We have averaged the Stromgren $b$ and $y$ band measurements from T10 to create a new $(b+y) / 2$ "passband" that gives us improved precision. T10 operation is very similar to our T8 $0.80 \mathrm{~m}$ APT, described in Henry $(1995,1999)$.

The T2 $\Delta V$ and T10 $\Delta(b+y) / 2$ observations are shown in the left and right panels, respectively, of Figure 2. Both data sets have been normalized so that all seasonal means agree with the mean of the first year, shown as the horizontal line in the two top panels. This removes any long-term brightness variability in the comparison stars and in HD 114762 itself; corrections were typically a few tenths of a mmag. The top panels of Figure 2 give for each APT the standard deviation of a single observation from the mean of the whole data set: $0.0122 \mathrm{mag}$ and $0.0012 \mathrm{mag}$ for T2 and T10, respectively. Both values are consistent with the precision of a single observation from each telescope, establishing the order of magnitude better precision of T10 over T2. Thus, we find that HD 114762 is constant to high precision on both night-to-night and year-toyear timescales.

The middle panels of Figure 2 plot the two data sets with respect to the orbital phase of the companion, with phase 0.0 equal to the predicted time of mid-transit, $T_{c}$, calculated from our revised orbital parameters in Table 3. A least-squares sine fit to each data set gives semi-amplitudes of $0.0011 \pm 0.0004$ and $0.00004 \pm 0.00005 \mathrm{mag}$. This confirms to very high precision the lack of stellar activity that might otherwise mimic periodic RV variations in HD 114762 (see, e.g., Queloz et al. 2001; Paulson et al. 2004; Bonfils et al. 2007; Forveille et al. 2009).

The bottom panels show the portion of the middle panels around phase 0.0 plotted with an expanded scale on the $x$ axis. The solid curve in each panel shows the predicted time $\left(T_{c}\right)$, depth $(\sim 1 \%)$, and duration (0.49 days) of a central transit, computed from the new orbital and planetary parameters derived above. The horizontal error bar below the transit shows the $\pm 1 \sigma$ uncertainty in $T_{c}$. With the present RV data set in this Letter, the uncertainty in $T_{c}$ is almost exactly equal to the transit duration. For the T2 observations in the bottom left panel of Figure 2, the mean of the 12 in-transit observations agrees with the mean of the 1977 out-of-transit observations to $0.0001 \pm 0.0035 \mathrm{mag}$, where the uncertainty is calculated by adding in quadrature the uncertainty of each of the two means. The uncertainty of the in-transit mean level dominates the total uncertainty because of 

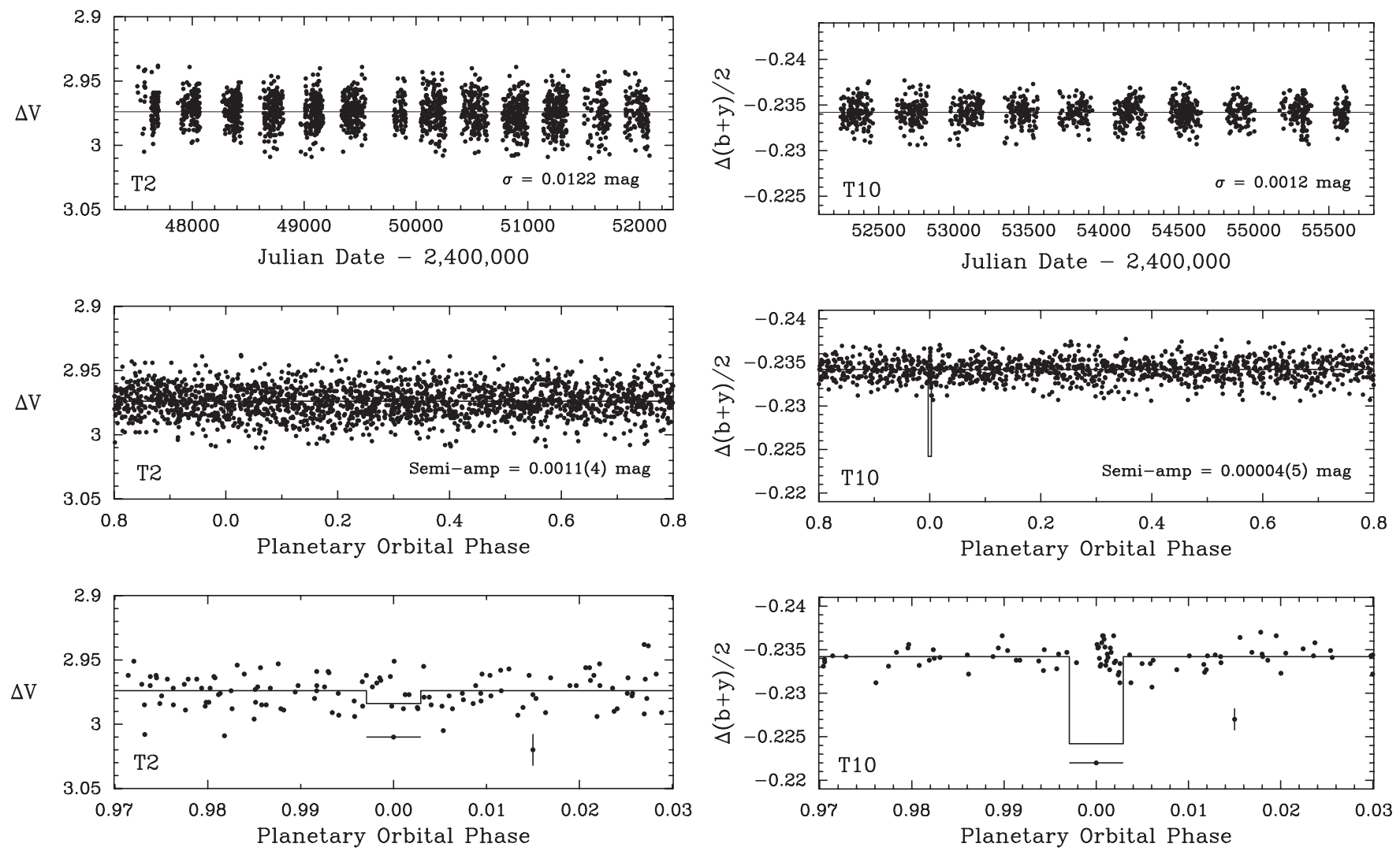

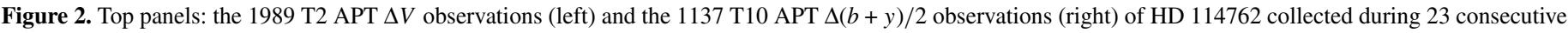

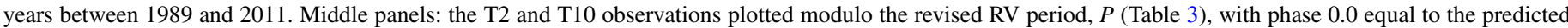

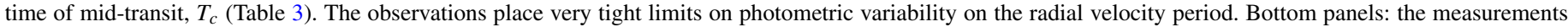

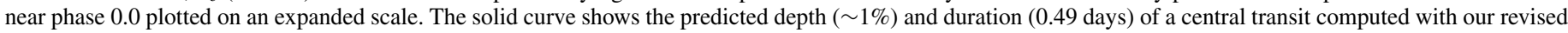

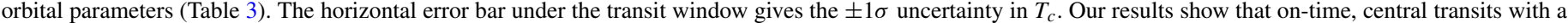
depth of $0.001 \mathrm{mag}$ or more do not occur.

the relatively fewer observations in transit than out. We have thus measured the difference between the in-transit and out-oftransit light levels to a precision of $0.0035 \mathrm{mag}$. If we assume the need for a $3 \sigma$ event to ensure detection, the T2 observations can only rule out transits $0.0105 \mathrm{mag}(\sim 1 \%)$ or deeper. Therefore, the $\mathrm{T} 2$ observations have just enough sensitivity to detect the predicted transits but fail to do so.

For the much more precise T10 observations in the bottom right panel of Figure 2, the difference between the mean of the 26 in-transit observations and the mean of the 1111 out-oftransit observations is $0.0001 \pm 0.0003 \mathrm{mag}$. Therefore, transits with a $3 \sigma$ depth of 0.0009 mag or more should be detectable. We round this to $0.001 \mathrm{mag}$ and note that this limit applies only to full-duration, on-time events. We must make this distinction because of the non-random distribution of observations in the predicted transit window. We have only one observation very early in the first half of the transit window and 25 observations that span the second half. Twenty-two of these 25 points came from a single night (JD 2455326) when we monitored HD 114762 at a higher cadence. The worst case for the detection of full-duration transits occurs if they are 1 standard deviation early, i.e., the window slides leftward one-half of its width. In this case, we loose all of the monitoring observations from the window and pick up only five nightly observations. With only $\frac{1}{4}$ the number of observations in the transit window, the $3 \sigma$ transit depth limit doubles to $0.002 \mathrm{mag}$. Thus, we can confidently rule out early or late full-duration transits to a limit of 0.002 mag. If the companion does transit as described, this is equivalent to ruling out radii of $0.44 R_{J}$, or a density of $86 \mathrm{~g} \mathrm{~cm}^{-3}$. For comparison, the density of the planet HATP-20b, with a mass of $7.246 M_{J}$ and a radius of $0.867 R_{J}$, is $13.8 \mathrm{~g} \mathrm{~cm}^{-3}$ (Bakos et al. 2011).

It is also worth considering transits whose trajectory lies along a chord of the star approximately half the stellar diameter. An on-time transit along such a chord would contain half the number of observations, all during the second half of the shorter transit. Thus, the detectable limit of an on-time transit with $\frac{1}{2}$ the full duration increases from $0.001 \mathrm{mag}$ to $\sim 0014 \mathrm{mag}$. The worst case for detection of these shorter transits occurs if they are $\frac{1}{2} \sigma$ early. In this case, the transit window contains only one observation, namely, the first observation in the transit window in Figure 2. The standard deviation of a single observation is $0.0012 \mathrm{mag}$, so the $3 \sigma$ depth limit for this worst case is $\sim 0.0036$ mag. But this worst case is very unlikely and happens only for $T_{c}$ exactly $\frac{1}{2} \sigma$ early. A little earlier or later, and the transit window begins picking up additional observations, and the depth limit becomes tighter. Therefore, we can exclude transits with $\frac{1}{2}$ full duration to a limit of $\sim 0.003$ mag. Similarly to that described above, this rules out transits of companions with densities lower than $47 \mathrm{~g} \mathrm{~cm}^{-3}$.

The T10 observations in the lower-right panel illustrate the utility of the TERMS approach to finding transits of planets with intermediate orbital periods. Of the 26 in-transit observations, only four of the nightly observations taken over 13 observing seasons fell at random within the transit window. The other 22 in-transit observations were acquired in a single night, 
2010 May 10 UT, predicted from our updated orbit based on our new RVs acquired within the TERMS program.

\section{CONCLUSIONS}

These new RV data refine the orbit of the first planetary candidate discovered to orbit a solar-type star. Our analysis of Lick spectra has greatly improved the estimated parameters of the host star. Including a linear trend into the Keplerian orbital fit does not significantly improve the rms scatter of the residuals and we thus find no evidence of any additional companions in the system. From the Keplerian fit we produce a refined transit ephemeris onto which we have folded 23 years of precise photometry acquired with the APT at Fairborn Observatory. Our photometric observations rule out on-time central transits to a limit of $\sim 0.001 \mathrm{mag}$, early or late central transits to a limit of $\sim 0.002 \mathrm{mag}$, and transits of $\frac{1}{2}$ full duration to a limit of $\sim 0.003 \mathrm{mag}$. Transits shorter than $\frac{1}{2}$ full duration are possible - they could hide in the gap in our transit coverage - but are highly unlikely due to the low probability of the required inclination angles and the exact timing needed to fit the transit within the gap. The absence of planetary transits constrains the orbital inclination to be less than $89^{\circ}$. , which raises the minimum mass by a negligible amount. For the companion to transit undetected for these data, the density would need to be in excess of $47 \mathrm{~g} \mathrm{~cm}^{-3}$, which is well outside the expected range for the planetary and brown dwarf regimes. The absolute confirmation of the planetary nature of the companion will likely need to await precision astrometric observations of this target to determine the orbital inclination.

The authors thank David Latham for useful comments on the manuscript. We also thank the anonymous referee, whose comments greatly improved the quality of the Letter. The Center for Exoplanets and Habitable Worlds is supported by the Pennsylvania State University, the Eberly College of Science, and the Pennsylvania Space Grant Consortium. G.W.H. acknowledges long-term support from NASA, NSF, Tennessee
State University, and the State of Tennessee through its Centers of Excellence program.

\section{REFERENCES}

Bakos, G. A., et al. 2011, ApJ, submitted (arXiv:1008.3388)

Bodenheimer, P., Laughlin, G., \& Lin, D. N. C. 2003, ApJ, 592, 555

Bonfils, X., et al. 2007, A\&A, 474, 293

Bowler, B. P., Liu, M. C., \& Cushing, M. C. 2009, ApJ, 706, 1114

Butler, R. P., et al. 2006, ApJ, 646, 505

Cochran, W. D., Hatzes, A. P., \& Hancock, T. J. 1991, ApJ, 380, L35

Demarque, P., Woo., J., Kim, Y., \& Yi, S. K. 2004, ApJS, 155, 667

Fabrycky, D. C., \& Winn, J. N. 2009, ApJ, 696, 1230

Forveille, T., et al. 2009, A\&A, 493, 645

Freedman, D. A. 1981, Ann. Stat., 9, 1218

Hale, A. 1995, PASP, 107, 22

Henry, G. W. 1995, in ASP Conf. Ser. 79, Robotic Telescopes: Current Capabilities, Present Developments, and Future Prospects for Automated Astronomy, ed. G. W. Henry \& J. A. Eaton (San Francisco, CA: ASP), 44

Henry, G. W. 1999, PASP, 111, 845

Henry, G. W., Baliunas, S. L., Donahue, R. A., Soon, W. H., \& Saar, S. H. 1997, ApJ, 474, 503

Howard, A. W., et al. 2010, ApJ, 721, 1467

Kane, S. R., Mahadevan, S., von Braun, K., Laughlin, G., \& Ciardi, D. R. 2009, PASP, 121, 1386

Kane, S. R., \& von Braun, K. 2008, ApJ, 689, 492

Knutson, H. A., Charbonneau, D., Cowan, N. B., Fortney, J. J., Showman, A. P., Agol, E., \& Henry, G. W. 2009a, ApJ, 703, 769

Knutson, H. A., et al. 2009b, ApJ, 690, 822

Latham, D. W., Mazeh, T., Stefanik, R. P., Mayor, M., \& Burki, G. 1989, Nature, 339,38

Mugrauer, M., Neuhäuser, R., Mazeh, T., Guenther, E., Fernández, M., \& Broeg, C. 2006, Astron. Nachr., 327, 321

Patience, J., et al. 2002, ApJ, 581, 654

Paulson, D. B., Saar, S. H., Cochran, W. D., \& Henry, G. W. 2004, AJ, 127, 1644

Percy, J. R., Bakos, A. G., \& Henry, G. W. 2000, PASP, 112, 840

Queloz, D., et al. 2001, A\&A, 379, 279

Robinson, E. L., Cochran, A. L., Cochran, W. D., \& Shafter, A. W. 1990, AJ, 99, 672

Valenti, J. A., \& Fischer, D. A. 2005, ApJS, 159, 141

Valenti, J. A., \& Piskunov, N. 1996, A\&AS, 118, 595

Valenti, J. A., et al. 2009, ApJ, 702, 989

van Leeuwen, F. 2007, A\&A, 474, 653

Wright, J. T., \& Howard, A. W. 2009, ApJS, 182, 205 